\title{
Erratum: Chaos and entanglement spreading in a non-commutative gauge theory
}

\author{
Willy Fischler, ${ }^{a}$ Viktor Jahnke ${ }^{b}$ and Juan F. Pedraza ${ }^{c}$ \\ ${ }^{a}$ Theory Group, Department of Physics and Texas Cosmology Center, \\ The University of Texas at Austin, Austin, TX 78712, U.S.A. \\ ${ }^{b}$ Departamento de Fúsica de Altas Energias, Instituto de Ciencias Nucleares, \\ Universidad Nacional Autónoma de México, Apartado Postal 70-543, CDMX 04510, Mexico \\ ${ }^{c}$ Institute for Theoretical Physics, University of Amsterdam, \\ Science Park 904, 1098 XH Amsterdam, Netherlands \\ E-mail: fischler@physics.utexas.edu, \\ viktor.jahnke@correo.nucleares.unam.mx, jpedraza@uva.nl
}

ERRATUM TO: JHEP11(2018)072

ABSTRACT: We point out minor mistakes appearing in the published version of our paper [1]. The main conclusions remain unaffected.

ArXiv EPrint: 1808.10050 
After the publication of the paper [1] minor errors were found, specifically, in the computation of the butterfly velocity.

First, in section 3 we forgot to take into account the effects of the compact space. To compensate for this fact, we can let the indices in equation (3.1) take values $i, j=1,2, \ldots 9$, where the additional coordinates, $x^{i}$ with $i=4,5, \ldots, 9$, represent the coordinates on the $S^{5}$. We also need to take $k^{i}=0$ for $i=4,5 \ldots, 9$, in equation (3.10), so the shockwave has momentum only along the boundary coordinates. With these changes, and noticing that the metric on the $S^{5}$ can be written as $G_{\theta \theta}(r) d \Omega_{5}^{2}$, we need to introduce the following changes:

- Equation (3.21) should now read:

$$
M^{2}=\left(\frac{2 \pi}{\beta}\right)^{2} \frac{G^{i i}\left(r_{\mathrm{H}}\right) G_{i i}^{\prime}\left(r_{\mathrm{H}}\right)}{G_{t t}^{\prime}\left(r_{\mathrm{H}}\right)}=\left(\frac{2 \pi}{\beta}\right)^{2} \frac{1}{G_{t t}^{\prime}\left(r_{\mathrm{H}}\right)}\left[\frac{G_{11}^{\prime}}{G_{11}}+2 \frac{G_{22}^{\prime}}{G_{22}}+5 \frac{G_{\theta \theta}^{\prime}}{G_{\theta \theta}}\right] .
$$

- Equation (3.27) should be replaced by:

$$
v_{B}^{2}(\phi)=\left.\frac{G_{t t}^{\prime}}{\left(2 \frac{G_{22}^{\prime}}{G_{22}}+\frac{G_{11}^{\prime}}{G_{11}}+5 \frac{G_{\theta \theta}^{\prime}}{G_{\theta \theta}}\right)} \frac{1}{G_{22} \sin ^{2} \phi+G_{11} \cos ^{2} \phi}\right|_{r=r_{\mathrm{H}}} .
$$

Therefore, the correct version of equations (3.28) and (3.29) must be:

$$
v_{B, x_{1}}^{2} \equiv v_{B}^{2}(\phi=0)=\left.\frac{G_{t t}^{\prime}}{G_{11}\left(2 \frac{G_{22}^{\prime}}{G_{22}}+\frac{G_{11}^{\prime}}{G_{11}}+5 \frac{G_{\theta \theta}^{\prime}}{G_{\theta \theta}}\right)}\right|_{r=r_{\mathrm{H}}},
$$

and

$$
v_{B, x_{2}}^{2} \equiv v_{B}^{2}(\phi=\pi / 2)=\left.\frac{G_{t t}^{\prime}}{G_{22}\left(2 \frac{G_{22}^{\prime}}{G_{22}}+\frac{G_{11}^{\prime}}{G_{11}}+5 \frac{G_{\theta \theta}^{\prime}}{G_{\theta \theta}}\right)}\right|_{r=r_{\mathrm{H}}},
$$

respectively.

- The final result for the components of the butterfly velocity, equations (3.30) and (3.31) should now be:

$$
v_{B}^{2}(\phi=0)=v_{B, x_{1}}^{2}=\frac{2}{3},
$$

and

$$
v_{B}^{2}(\phi=\pi / 2)=v_{B, x_{2}}^{2}=\frac{2}{3}\left(1+a^{4} r_{\mathrm{H}}^{4}\right) .
$$

In summary, the component of the butterfly velocity along the commutative $x_{1}$-direction turns out to be independent on $a r_{\mathrm{H}}$, taking the universal conformal value, i.e., $v_{B, x_{1}}^{2}=2 / 3$. On the other hand, the components along the non-commutative plane approach the conformal value only in the IR, $v_{B, x_{2}}^{2}\left(a r_{\mathrm{H}} \rightarrow 0\right) \rightarrow 2 / 3$, and grow monotonically as $a r_{\mathrm{H}}$ is increased. The final formula for these components differs from the one previously reported, however, all of the qualitative features and main conclusions remain the same (for these components). We also note that figure 1 in the paper should be replaced by the figure below: 


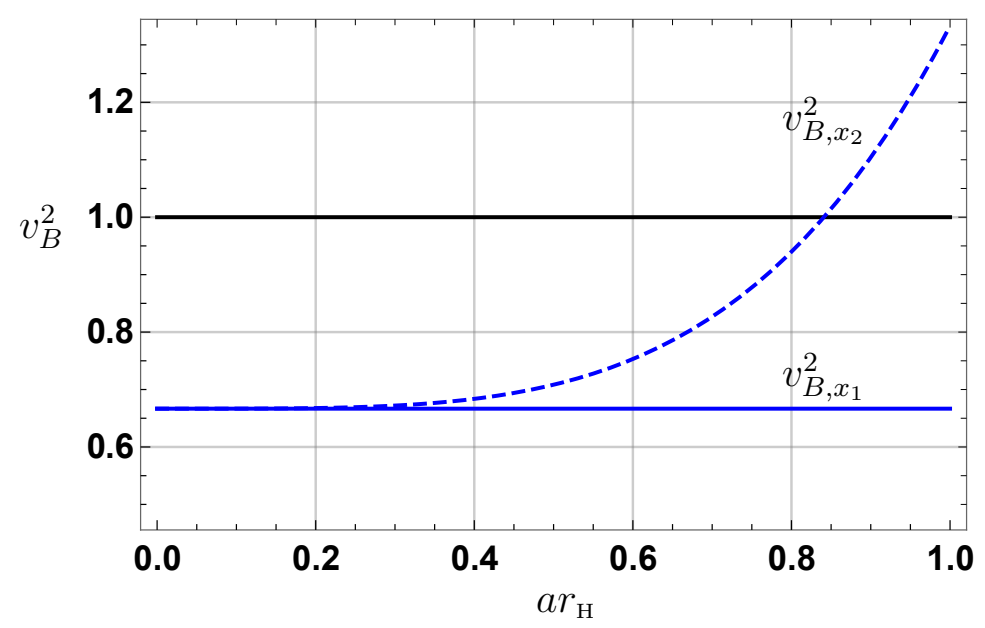

Figure 1. Butterfly velocity squared $v_{\mathrm{B}}^{2}$ versus the dimensionless parameter $a r_{\mathrm{H}}$. The horizontal blue line represents the butterfly velocity along the $x_{1}$-direction, which does not depend on $a r_{\mathrm{H}}$ and takes the conformal value $v_{\mathrm{B}}^{2}=2 / 3$, while the dashed blue curve represents the butterfly velocity along the $x_{2}$ - and $x_{3}$-directions. The horizontal black line corresponds to the speed of light.

The section 5 requires similar modifications. In equation (5.1) we must let the indices take values $i, j=1,2, \ldots, D$, where the extra coordinates $x^{i}$ with $i=d, d+1, \ldots, D$ describe the compact space, $G_{\theta \theta}(r) d \Omega_{5}^{2}$ in our case. With this in mind, equation (5.8) should now read:

$$
M^{2}=\left(\frac{2 \pi}{\beta}\right)^{2} \frac{G^{i i}\left(r_{\mathrm{H}}\right) G_{i i}^{\prime}\left(r_{\mathrm{H}}\right)}{G_{t t}^{\prime}\left(r_{\mathrm{H}}\right)}=\left(\frac{2 \pi}{\beta}\right)^{2} \frac{1}{G_{t t}^{\prime}\left(r_{\mathrm{H}}\right)}\left[\frac{G_{11}^{\prime}}{G_{11}}+2 \frac{G_{22}^{\prime}}{G_{22}}+5 \frac{G_{\theta \theta}^{\prime}}{G_{\theta \theta}}\right] .
$$

The rest of the section is unchanged. In particular, equation (5.15) yields the right answer for the components of the butterfly velocity provided that the sum over $k$ runs over all coordinates, including those in the compact space. Indeed, it can be checked that with this formula we can recover the results that reported above using the shockwave method, equations (5) and (6), respectively.

Open Access. This article is distributed under the terms of the Creative Commons Attribution License (CC-BY 4.0), which permits any use, distribution and reproduction in any medium, provided the original author(s) and source are credited.

\section{References}

[1] W. Fischler, V. Jahnke and J.F. Pedraza, Chaos and entanglement spreading in a non-commutative gauge theory, JHEP 11 (2018) 072 [arXiv:1808.10050] [INSPIRE]. 